Acta Crystallographica Section D

Biological Crystallography

ISSN 0907-4449

\section{Purification, crystallization and preliminary X-ray analysis of haemoglobin I from the armoured catfish Liposarcus anisitsi}

Received 31 March 1999 Accepted 1 February 2000

\author{
A. L. S. Smarra, ${ }^{a}$ W. F. de \\ Azevedo Jr, ${ }^{a}$ V. Fadel, ${ }^{a, c, d}$ P. \\ Delatorre, ${ }^{a} M$. Dellamano, ${ }^{a} M$. F. \\ Colombo $^{a}$ and G. O. Bonilla- \\ Rodriguez $^{b_{*}}$
}

${ }^{\mathrm{a}}$ Departamento de Física, Instituto de Biociências, Letras e Ciências Exatas, UNESP, São José do Rio Preto, SP 15054-000, Brazil, ${ }^{\mathbf{b}}$ Departamento de Química e Geociências, Instituto de Biociências, Letras e Ciências Exatas, UNESP, São José do Rio Preto, SP 15054-000, Brazil, ' Departamento de Física, Faciba-FEB, Barretos, SP 14783-226, Brazil, and ${ }^{\mathbf{d}}$ Centro Universitário de Votuporanga, CEUV, Votuporanga, SP 15500-030, Brazil

Correspondence e-mail: bonilla@qeg.ibilce.unesp.br
C) 2000 International Union of Crystallography Printed in Denmark - all rights reserved

\begin{abstract}
Liposarcus anisitsi is an armoured catfish that presents accessorial air oxygenation through a modified stomach, which allows this species to survive in waters with very low oxygen content. Analysis of its haemolysate has shown the presence of four haemoglobins; this work focuses on the main component, haemoglobin I. It has been crystallized in two different forms and X-ray diffraction data have been collected to 2.77 and $2.86 \AA$ resolution using synchrotron radiation. Crystals were determined to belong to the space groups $C 2$ and $P 2_{1}$ and preliminary structural analysis revealed the presence of one tetramer in the asymmetric unit in both crystal forms. The structure was determined using a standard molecular-replacement technique.
\end{abstract}

\section{Introduction}

Haemoglobin functions in aerobic organisms by dramatically increasing oxygen diffusion from the environment (aquatic or atmospheric) to its reduction site at the inner mitochondrial membrane. Aerobic metabolism in multicellular organisms would be impossible without this protein.

Owing to its abundance and availability, haemoglobin has been largely used as a model in order to gain understanding of oligomeric proteins, particularly concerning the heterotropic 'fine tuning' of oxygen binding in terms of affinity and cooperativity (Smarra et al., 1999). Recent reports describing 'new' structures found for human adult haemoglobin (Smith et al., 1991; Mercedes-Silva et al., 1992; Seixas et al., 1999) additional to those considered by the classical allosteric model of Monod et al. (1965) have revealed new aspects, despite the large amount of research spent on the subject.

The study of the structures of other forms of haemoglobin could contribute to providing a better understanding of haemoglobin function. The animal kingdom has plenty of isomorphic haemoglobins working under a variety of physiological requirements and subject to environmental stress, being a natural source for the study of structure-function relationships.

L. anisitsi (Siluriformes, Loricariidae) is a South American armoured catfish with accessorial air oxygenation using a modified stomach. This morphological and histological adaptation allows the fish to hold swallowed air and oxygenate blood circulating on abundant capillaries close to the mucosa (Smarra et al.,
1997). Its blood has four haemoglobins, with isoelectric points (pI) in the range 7.2-8.3. These proteins were denominated I to IV according to their pI, starting with the highest value.

The present work was performed on the oxygenated form of $\mathrm{Hb}$-I (which comprises about $50 \%$ of the haemolysate). Structural studies have also been initiated on the unliganded forms.

\section{Materials and methods}

\subsection{Purification}

Blood was collected by caudal puncture after an adaptive period of $4 \mathrm{~d}$ in aquaria. Subsequent procedures were carried out at low temperature (around $277 \mathrm{~K}$ ). Red blood cells were washed by centrifugation four times with buffered saline $(50 \mathrm{~m} M$ Tris $\mathrm{pH}$ 8.5, $1 \mathrm{~m} M$ EDTA). Haemolysis was accomplished with buffer $A$ (50 m $M$ Tris pH 8.5, $1 \mathrm{~m} M$ EDTA), followed by centrifugation ( $1000 \mathrm{~g}$ for $1 \mathrm{~h}$ ) and filtration to remove debris. An aliquot was kept for phosphate determination. For phosphate removal, haemolysate dialysis was performed against buffer $A$, followed by gel filtration (Sephadex G-25) on a $1.6 \times 70 \mathrm{~cm}$ column.

Haemoglobin purification was performed on DEAE-Sephadex A-50 using a $\mathrm{pH}$ gradient between $50 \mathrm{~m} M$ Tris buffer $\mathrm{pH} 9.2$ and $50 \mathrm{mM}$ bis-Tris $\mathrm{pH}$ 6.5. The isolated components were further deionized by several passages through a mixed-bed Amberlite MB-1 (Sigma), concentrated by centrifugation on Amicon microconcentrators and stored in liquid nitrogen until use. 
Table 1

Detailed X-ray diffraction statistics for synchrotron data of crystal form I.

\begin{tabular}{llcllc}
\hline $\begin{array}{l}\text { Resolution } \\
\text { range }(\AA)\end{array}$ & $\begin{array}{l}\text { Number of } \\
\text { independent } \\
\text { reflections }\end{array}$ & $I / \sigma(I)$ & $\begin{array}{l}\text { Complete- } \\
\text { ness, all } \\
\text { data }(\%)\end{array}$ & $\begin{array}{l}\text { Redun- } \\
\text { dancy }\end{array}$ & $\begin{array}{l}R_{\text {merge }}{ }^{\dagger} \text {, } \\
\text { all data } \\
(\%)\end{array}$ \\
\hline $15.00-6.05$ & 1100 & 18.2 & 85.6 & 2.62 & 4.0 \\
$6.05-4.78$ & 1208 & 17.6 & 95.0 & 2.61 & 4.4 \\
$4.78-4.16$ & 1186 & 18.0 & 93.8 & 2.60 & 5.5 \\
$4.16-3.77$ & 1226 & 16.5 & 93.5 & 2.20 & 6.6 \\
$3.77-3.49$ & 1237 & 16.3 & 92.2 & 2.34 & 6.4 \\
$3.49-3.28$ & 1263 & 14.2 & 93.1 & 2.50 & 7.0 \\
$3.28-3.12$ & 1261 & 10.4 & 92.6 & 2.40 & 8.1 \\
$3.12-2.98$ & 1235 & 9.3 & 90.2 & 2.33 & 12.0 \\
$2.98-2.86$ & 1294 & 7.0 & 87.3 & 2.22 & 14.6 \\
$2.86-2.77$ & 1304 & 5.8 & 78.9 & 1.90 & 20.1 \\
All reflec- & 12314 & 13.3 & 90.2 & 2.37 & 8.9 \\
tions & & & & & \\
\hline
\end{tabular}

$\dagger R_{\text {merge }}=100 \sum_{h k l}\left[\sum_{i}\left(\left|I_{h k l, i}-\left\langle I_{h k l}\right\rangle\right|\right)\right] / \sum_{h k l, i}\left(I_{h k l}\right)$, where $I_{h k l, i}$ is the intensity of an individual measurement of the reflection with indices $h \mathrm{kl}$ and $\left\langle I_{h k l}\right\rangle$ is the mean intensity of that reflection.

Table 2

Detailed X-ray diffraction statistics for synchrotron data of crystal form II.

\begin{tabular}{llcllc}
\hline $\begin{array}{l}\text { Resolution } \\
\text { range }(\AA)\end{array}$ & $\begin{array}{l}\text { Number of } \\
\text { independent } \\
\text { reflections }\end{array}$ & $I / \sigma(I)$ & $\begin{array}{l}\text { Complete- } \\
\text { ness, all } \\
\text { data (\%) }\end{array}$ & $\begin{array}{l}\text { Redun- } \\
\text { dancy }\end{array}$ & $\begin{array}{l}R_{\text {merge }} \dagger \\
\text { all data } \\
(\%)\end{array}$ \\
\hline $15.00-6.15$ & 1095 & 18.4 & 74.5 & 2.47 & 6.6 \\
$6.15-4.89$ & 1202 & 16.8 & 81.6 & 2.47 & 8.4 \\
$4.89-4.27$ & 1180 & 17.2 & 82.6 & 2.39 & 7.7 \\
$4.27-3.88$ & 1220 & 15.7 & 84.3 & 2.42 & 8.6 \\
$3.88-3.60$ & 1230 & 12.8 & 86.0 & 2.38 & 9.7 \\
$3.60-3.39$ & 1255 & 10.4 & 86.9 & 2.34 & 11.7 \\
$3.39-3.22$ & 1255 & 8.1 & 87.4 & 2.35 & 13.8 \\
$3.22-3.08$ & 1231 & 6.5 & 88.0 & 2.30 & 17.4 \\
$3.08-2.96$ & 1291 & 5.4 & 88.9 & 2.30 & 19.0 \\
$2.96-2.86$ & 1290 & 4.4 & 90.0 & 2.29 & 22.0 \\
All reflec- & 12249 & 11.4 & 85.0 & 2.37 & 8.3 \\
tions & & & & & \\
\hline
\end{tabular}

$\dagger R_{\text {merge }}=100 \sum_{h k l}\left[\sum_{i}\left(\left|I_{h k l, i}-\left\langle I_{h k l}\right\rangle\right|\right)\right] / \sum_{h k l, i}\left(I_{h k l}\right)$, where $I_{h k l, i}$ is the intensity of an individual measurement of the reflection with indices $h k l$ and $\left\langle I_{h k l}\right\rangle$ is the mean intensity of that reflection. silicate glass capillary tube for $\mathrm{X}$-ray data collection.

\subsection{X-ray data collection and processing}

Two crystal forms had their X-ray diffraction data sets collected at a wavelength of $1.38 \AA$ using the Synchrotron Radiation Source [Station PCr, Laboratório Nacional de Luz Síncrotron (LNLS), Campinas, Brazil; Polikarpov, Oliva et al., 1998; Polikarpov, Perles et al., 1998] and a $34.5 \mathrm{~cm}$ MAR imaging-plate detector (MAR Research). X-ray diffraction data for crystal forms I and II were processed to 2.77 and $2.86 \AA$, respectively, using the program $D E N Z O$ and were scaled using the program SCALEPACK (Otwinowski, 1993).

Autoindexing procedures combined with analysis of the $\mathrm{X}$-ray diffraction pattern and averaging of equivalent intensities were used in the characterization of the Laue symmetry for both crystals.

\subsection{Molecular replacement}

The crystal structure of the oxyhaemoglobin I isolated from L. anisitsi was determined by standard molecular-replacement methods using the program AMoRe (Navaza, 1994). The atomic coordinates of the dimer $(\alpha \beta)$ of trout haemoglobin

\subsection{Crystallization}

The oxygenated form of haemoglobin I (LaHb-I) was concentrated to $10 \mathrm{mg} \mathrm{ml}^{-1}$ in $50 \mathrm{~m} M$ Tris $\mathrm{pH}$ 8.5. Crystallization trials were performed using the hanging-drop vapour-diffusion method (Jancarik \& Kim, 1991).

Crystals of L. anisitsi haemoglobin have been obtained under several different crystallization conditions using the hanging-drop vapor-diffusion and sparse-matrix methods (Smarra et al., 1997). The best crystals were obtained after $3 \mathrm{~d}$ from drops in which $7 \mu \mathrm{l}$ haemoglobin solution $\left(10 \mathrm{mg} \mathrm{ml}^{-1}\right)$ was mixed with an equal volume of reservoir solution. 0.1 $M$ HEPES pH 7.4, $1.4 M$ sodium citrate was used as the reservoir solution. The crystals were harvested in the well buffer and mounted in a $1.0 \mathrm{~mm}$ boro- deposited in the PDB (Tame et al., 1996; PDB code 1ouu) were used as a search model. All water and phosphate molecules were removed from the search model and the temperature factors for all atoms were set to $20 \AA^{2}$; however, the haem groups were retained in the model. The atomic coordinates for the search model were translated to set their centre of gravity at the origin and were then rotated so that the principal axes of inertia of the search model lie parallel to orthogonal axes.

Cross-rotation functions were calculated in the resolution range $10-4.5 \AA$ with a sampling step of $2.5^{\circ}$ using the program AMoRe (Navaza, 1994). These calculations were carried out with an integration radius of $25 \AA$. The rotation which generated the highest correlation coefficient (CC) was applied to the search model and used in the subsequent translation-function computations based on data in the same resolution range.

\section{Results and discussion}

LaHb-I crystallized in two different space groups under the same crystallization conditions. Crystal form I belongs to the monoclinic $C 2$ Bravais lattice, with unit-cell parameters $a=57.2(1), b=62.9$ (1), $c=183.4$ (2) $\AA, \beta=92.6$ (2) . Assuming the asymmetric unit content to be one tetramer of molecular weight $65 \mathrm{kDa}$, the $V_{m}$ value is $2.5 \AA^{3} \mathrm{Da}^{-1}$ (Matthews, 1968). Assuming a value of $0.74 \mathrm{~cm}^{3} \mathrm{~g}^{-1}$ for the protein partial specific volume, the calculated solvent content in the crystal is $51.5 \%$ and the calculated crystal density is $1.175 \mathrm{~g} \mathrm{~cm}^{-3}$. A summary of the X-ray data-collection statistics is given in Table 1.

Crystal form II also belongs to the monoclinic system; however, it has different unit-cell parameters $a=56.8$ (1), $b=64.0$ (1), $c=86.7$ (2) $\AA, \beta=92.2^{\circ}$. The observed systematic absences are compatible with space group $P 2_{1}$. Assuming that one tetramer is present in an asymmetric unit, the $V_{m}$ value is $2.4 \AA^{3} \mathrm{Da}^{-1}$. The solvent content in the crystal is $48.8 \%$ and the calculated crystal density is $1.184 \mathrm{~g} \mathrm{~cm}^{-3}$. Table 2 shows the results for data collection from crystal form II.

The X-ray diffraction data for crystal form II were used in the molecular replacement. The highest magnitude of the correlation function was obtained for the Euler angles $\alpha$ $=258.02, \beta=121.82, \gamma=127.00^{\circ}$ for the first dimer $\left(\alpha_{1} \beta_{1}\right)$ and angles $\alpha=78.53, \beta=64.15$, $\gamma=139.52^{\circ}$ for the second dimer $\left(\alpha_{2} \beta_{2}\right)$. The correlation coefficient and the $R$ factor after the translation search were 67.1 and $36.6 \%$, respectively. The fractional coordinates are $T_{x}=0.2368, T_{y}=0.0010, T_{y}=0.1707$ for the first dimer and $T_{x}=0.3460, T_{y}=0.3549$, $T_{z}=0.3191$ for the second dimer. The X-ray diffraction data for crystal form I were also used in the molecular replacement; however, a lower correlation coefficient $(52.4 \%)$ and a higher $R$ factor $(44.3 \%)$ were generated after the translation search using the same search model; therefore, X-ray data for crystal form II were used in the preliminary refinement.

Repeated cycles of refinement were carried out using the maximum-likelihood method as implemented in the program REFMAC (Murshudov et al., 1997). The present values of $R$ and $R_{\text {free }}$ are 27.2 and $36.9 \%$, respectively, using the sequence of trout haemoglobin (PDB code 1ouu). Amino-acid sequencing for LaHb-I using 
the automated Edman technique is under way. Further refinement will be carried out as soon as the sequence become available.

We thank Dr P. Kuser and Dr I. Polikarpov (LNLS) for their help in the synchrotron data collection. The authors also thank $\mathrm{Dr}$ Waldener Garutti and $\mathrm{Dr}$ Francisco Langeani Neto, who kindly supplied specimens from the Centro de Aqüicultura (Caunesp-Unesp). This work was supported by grants from FAPESP, CNPq, CAPES and FUNDUNESP (Brazil).

\section{References}

Jancarik, J. \& Kim, S.-H. (1991). J. Appl. Cryst. 24 409-411.

Matthews, B. W. (1968). J. Mol. Biol. 33, 491-497.

Mercedes Silva, M., Rogers, P. H. \& Arnone, A. (1992). J. Biol. Chem. 276, 17248-17256.

Monod, J., Wyman, J. \& Ghangeux, J. P. (1965). J. Mol. Biol. 12, 88-118.

Murshudov, G. N., Vagin, A. A. \& Dodson, E. J. (1997). Acta Cryst. D53, 240-255.

Navaza, J. (1994). Acta Cryst. A50, 157-163.

Otwinowski, Z. (1993). Proceedings of the CCP4 Study Weekend. Data Collection and Processing, edited by L. Sawyer, N. Isaacs \& S. Bailey, pp. 56-62. Warrington: Daresbury Laboratory.

Polikarpov, I., Oliva, G., Castellano, E. E., Garratt, R., Arruda, P., Leite, A. \& Craievich, A. (1998). Nucl. Instrum. Methods A, 405, 159-164.
Polikarpov, I., Perles, L. A., de Oliveira, R. T., Oliva, G., Castellano, E. E., Garratt, R. \& Craievich, A. (1998). J. Synchrotron Rad. 5, 72-76.

Seixas, F. A., de Azevedo, W. F. Jr \& Colombo, M. F. (1999). Acta Cryst. D55, 1914-1916.

Smarra, A. L. S, Arni, R. K., de Azevedo, W. F. Jr, Colombo, M. F. \& Bonilla-Rodriguez, G. O. (1997). Protein Pept. Lett. 4(5), 349-354.

Smarra, A. L. S., Fadel, V., Dellamano, M., Olivieri, J. R., de Azevedo, W. F. Jr \& BonillaRodriguez, G. O. (1999). Acta Cryst. D55, 1618-1619.

Smith, F. R., Lattman, E. E. \& Carter, C. W. Jr (1991). Proteins Struct. Funct. Genet. 10, 81-91.

Tame, J. R., Wilson, J. C. \& Weber, R. E. (1996). J. Mol. Biol. 259, 749-760. 